\title{
ON RINGS WITH QUASI-INJECTIVE CYCLIC MODULES
}

\author{
by J. AHSAN \\ (Received 30th May 1973)
}

\section{Introduction}

A ring $R$ is called a qc-ring if each cyclic $R$-module is quasi-injective. For various properties of these rings we refer to Ahsan (1) and Koehler (15). In this paper we shall obtain some additional results related to qc-rings. The scheme of the paper is as follows. Section 2 contains various preliminary definitions and results. In Section 3, we shall prove that every commutative hypercyclic ring is a qc-ring. In this section, we shall also show that a qc-ring which satisfies the ascending chain condition on its annihilators has nilpotent Jacobson-radical. Finally, in Section 4, we shall study rings all of whose proper factor rings are qc. Such rings will be called " restricted qc".

\section{Preliminaries}

Throughout this paper we shall assume that every ring has an identity and that every module is unitary. If $X$ is a subset of $R, r(X)(l(X))$ will denote the right (left) annihilator of $X$ in $R$. $J$ will denote the Jacobson-radical of a ring $R$. A ring is said to be bound to its radical if $l(J) \cap r(J) \subset J . \quad R$ is called local (semilocal) if $R / J$ is a division ring (semi-simple Artinian). It is known that in a semilocal ring $R$, the right (left) socle of $R$ is $l(J)(r(J))$ (see (18)). A ring $R$ is called a duo ring if every one-sided ideal of $R$ is two sided. A ring $R$ is semi-primary if $R / J$ is semi-simple Artinian and $J$ nilpotent. We shall also use some properties of uniserial and qF-rings. For different characterisations of these rings, we refer to Bass (3), Faith (9, 10), Faith and Walker (10), Asano (2), Fuller (12) and Byrd (5). If $P$ is any property of rings then a ring $R$ is restricted $P$ if every proper homomorphic image of $R$ has property $P$. Restricted qF (uniserial) rings have been studied by Levy (16), Faith (9), Pollinghar and Zaks (19) and Zaks (21). A ring $R$ is called a $q$-ring if each right ideal of $R$ is quasi-injective. The study of q-rings was initiated by Jain, Singh, and Mohamed (13). In (6), Caldwell called a ring $R$ hypercyclic if each cyclic right $R$-module has a cyclic injective hull. Caldwell proved that a perfect ring is hypercyclic if and only if it is uniserial. He has also shown, by means of an example, that an arbitrary hypercyclic ring need not satisfy any chain condition even in the commutative case. 


\section{Hypercyclic rings and quasi-injective modules}

We prove the following theorem.

Theorem 3.1. Let $R$ be a commutative ring. If $R$ is hypercyclic then $R$ is qc.

Proof. In order to prove that $R$ is a qc-ring, we need to show that each cyclic $R$-module is quasi-injective. Let $A$ be a cyclic $R$-module with injective envelope $E$. Since $R$ is hypercyclic, $E$ is cyclic. Let $g$ be a generator of $E$, i.e. $E=g R$. Let $\theta \in \Lambda=\operatorname{Hom}_{R}(E, E)$ and let $\theta(g)=g s$. Then if $a \in A$, we have $a=g r$ and $\theta(a)=\theta(g) r=g s r=g r s=a s$. Therefore, $\theta(a) \in A$. Hence by Johnson and Wong (14, Theorem 1.1) $A$ is quasi-injective. Therefore $R$ is qc.

We now give an example to show that arbitrary hypercyclic rings need not be qc.

Example 3.2. Let $R$ be the ring of integers $\bmod 4$ and $R_{2}$ the ring of all $2 \times 2$ matrices over $R$. Since $R$ is a uniserial ring, so also is $R_{2}$. Hence by Theorem 1.5 of Caldwell (6), $R_{2}$ is hypercyclic. By Theorem 8 of (1), $R_{2}$ is not a qc-ring.

The above example can also be used to support the statement that it is not necessary for a ring all of whose factor rings are self-injective to be a qc-ring. A commutative ring is uniserial if and only if all its homomorphic images are hypercyclic (see Caldwell (6, Theorem 2.5)). Since qc-rings are closed under the taking of homomorphic images (1, Lemma 3$)$ but need not be uniserial even in the commutative case (1, Example 2), it follows that a qc-ring need not be hypercyclic even if it is commutative.

We recall from (1) that a qc-ring is called strongly qc if its Jacobson-radical is nilpotent. A qc-ring is strongly $\mathrm{qc}$ if and only if it is Noetherian (see (1, Theorem 5)). In the next theorem, we show that for a qc-ring $R$ to be strongly qc, it is sufficient to assume that $R$ satisfies the ascending chain condition on right annihilators.

Theorem 3.3. Let $R$ be a ring. Then the following statements are equivalent:

(1) $R$ is a qc-ring which satisfies the ascending chain condition on right annihilators.

(2) $R$ is strongly qc.

(3) $R$ is a uniserial ring all of whose one-sided large ideals are two-sided.

Proof. $(1) \Rightarrow(2)$. Since $R$ is a qc-ring, $R_{R}$ is injective. Hence by Corollary 5 , page 47 of Faith (8), the right singular ideal of $R$ is equal to $J$. By our assumption, $R$ satisfies the ascending chain condition on right annihilators; hence by Corollary 8 on page 312 of Shock (20), $J$ is nilpotent. Therefore, $R$ is strongly qc.

$(2) \Leftrightarrow(3)$ follows from (1, Theorem 7).

$(2) \Rightarrow(1)$ is clear from the remark above. 


\section{Restricted qc-rings}

A ring $R$ will be called a restricted qc-ring if each proper factor ring of $R$ is a qc-ring. It is not necessary that a ring all of whose (proper) factor rings are self-injective is restricted qc (e.g. see Example 4.8 below). Simple rings are, trivially, restricted qc and thus a complete classification of restricted qc-rings seems difficult to obtain. We shall, however, obtain some important properties of these rings. We start with the following examples.

Example 4.4. The ring $Z$ of integers is restricted qc. Since every proper factor ring of $\mathbf{Z}$ is a (commutative) principal ideal Artinian ring and hence is uniserial, and also since every commutative uniserial ring is a qc-ring by Theorem 3.3, every proper factor ring of $\mathbf{Z}$ is qc. Therefore, $\mathbf{Z}$ is restricted qc. Clearly, $\mathbf{z}$ itself is not a qc-ring, as it is not self injective.

Example 4.5. Let $F$ be a field and $x$ an indeterminate over $F$. Let $W$ be the family of all well-ordered sets $\{I\}$ of non-negative real numbers, the order relation being the order of real numbers. Let $R$ be the set of all formal power series $\Sigma a_{i} x_{i}$ with $a_{i} \in F$ and $\{I\} \in W$. Then by (1, Example 2), $R$ is a restricted qc-ring which does not satisfy any chain condition.

We now prove the following theorem.

Theorem 4.6. Let $R$ be any ring with non-zero Jacobson-radical. If $R$ is restricted qc then $R$ is a direct sum of two rings $R_{1}$ and $R_{2}$, where $R_{1}$ is semisimple Artinian and $R_{2}$ is a semi-local ring bound to its radical.

Proof. Since $R$ is restricted qc and $\mathrm{J} \neq 0, R / J$ is a qc-ring. Therefore, $R / J$ is Artinian by Theorem 1 of (1). This means that $R$ is a semi-local ring. The theorem now follows from Mohamed (18, Theorem 1.5).

The following Corollary may be deduced from the above theorem by using the arguments in the proof of Theorem 2 of (1).

Corollary If $J$ is non-nilpotent then for each positive integer $n, J^{n} / J^{n+1}$ is a finite direct sum of simple $R$-modules.

Theorem 4.7. Let $R$ be a Noetherian ring. If $R$ is restricted qc then $R$ is restricted uniserial.

Proof. Let $I$ be a non-zero ideal of $R$. Since $R$ is restricted qc, $R / I$ is a qc-ring. This implies that $R / I$ is, in particular, a self-injective ring. $R / I$ is also Noetherian since $R$ is so. Therefore $R / I$ is an Artinian ring (see Lemma 7 of (1)). Hence $J(R / I)$ is nilpotent. Thus $R / I$ is a strongly qc-ring. Then it follows from Theorem 3.3 that $R / I$ is a uniserial ring. We have shown that every proper factor ring of $R$ is uniserial. Hence $R$ is restricted uniserial.

The following example shows that restricted uniserial rings need not be restricted qc.

Example 4.8. Let $Z_{n}$ be the ring of $n \times n$ matrices $(n>1)$ with entries from $\mathbf{Z}$, 
the ring of integers. Since $\mathbf{Z}$ is restricted uniserial, $\mathbf{Z}_{n}$ is also restricted uniserial. $\mathbf{Z}_{n}$ is not restricted qc. This is possible if and only if every proper factor ring of $\mathbf{Z}$ is semi-simple Artinian by Theorem 8 of (1).

Theorem 4.9. Let $R$ be a restricted qc-ring and $J \neq 0$. If $J$ is nilpotent then $R$ is restricted uniserial.

Proof. Since $R$ is restricted qc, $R / J$ is Artinian by Theorem 4.6. But $J$ is nilpotent. Hence $R$ is a semi-primary ring. Let $I$ be any non-zero ideal of $R$. Then $R / I$ is a qc-ring which is also semi-primary by a result of Björk ((4), Lemma 3.1). Thus $R / I$ is a strongly qc-ring, whence it is uniserial by Theorem 3.3. Therefore, $R$ is restricted uniserial.

Theorem 4.10. Let $R$ be a commutative ring. Then $R$ is restricted $q F$ (uniserial) if and only if $R$ is restricted $q c$ and Noetherian.

Proof. (1) Let us suppose that $R$ is restricted uniserial. Then each proper factor ring of $R$ is uniserial. This implies that each proper factor ring of $R$ is qc by Theorem 3.3, i.e. $R$ is restricted qc. Since $R$ is restricted uniserial, it is restricted Artinian. But a commutative restricted Artinian ring is always Noetherian by Cohen (7).

(2) Conversely, if $R$ is Noetherian and restricted qc, then it follows from Theorem 4.7 that $R$ is restricted uniserial. This completes the proof of the theorem.

We have already given the definition of q-rings. Every self-injective duo ring is a q-ring (see Jain, Singh and Mohamed (13)). It is not necessary that each proper homomorphic image of a Noetherian q-ring is also a q-ring (see the Example on page 209 of Faith (9)). In (17), Mohamed has given a partial classification of Noetherian rings whose proper homomorphic images are q-rings. Using the notion of restricted qc-rings, a complete classification of rings, among Noetherian rings, whose proper homomorphic images are q-rings, can be obtained as follows.

Theorem 4.11. Let $R$ be Noetherian. Then each proper homomorphic image of $R$ is a right q-ring if and only if $R$ is restricted $q c$.

Proof. (1) Let us suppose that each proper homomorphic image of $R$ is a q-ring. Let $I$ be a non-zero ideal of $R$. Then $R / I$ is a q-ring. Therefore $R / I$ is a self-injective ring. Since $R$ is Noetherian so also is $R / I . \quad R / I$ is, therefore, a qF-ring. Let $K / I$ be any ideal of $R / I$ where $K \varsubsetneqq I$. Then $R / I / K / I \cong R / K$. Because $K \neq(0)$, the fact that each proper homomorphic image of $R$ is a q-ring implies that $R / K$ is a q-ring so that $R / K$ is self-injective. However, $R / K$ is also Noetherian. Hence $R / K$ is a qF-ring. This means that $(R / I) /(K / I)$ is a $\mathrm{qF}$-ring so that every factor ring of $R / I$ is also $\mathrm{qF}$. Hence, by Fuller (11), $R / I$ is uniserial. By Theorem 11 of $(1)$, every uniserial q-ring is also qc. Hence $R / I$ is a qc-ring. We have shown that $R$ is restricted qc. 
(2) Conversely, let us suppose that $R$ is restricted qc. Since $R$ is Noetherian, it is restricted uniserial by Theorem 4.8. But every uniserial qc-ring is also a q-ring (1, Theorem 11). Hence $R$ is a restricted q-ring. This completes the proof of the theorem.

Theorem 4.12. Let $R$ be a right Noetherian ring. Then each homomorphic image of $R$ is a q-ring if and only if $R$ is uniserial and every large one sided ideal of $R$ is two sided.

Proof. (1) Let us suppose that $R$ is a Noetherian ring all of whose homomorphic images are q-rings. If $I$ is any ideal of $R$, then the factor ring $R / I$ is a $\mathrm{q}$-ring and hence self-injective. $R / I$ is also Noetherian. Therefore $R / I$ is a qF-ring. Since every homomorphic image of $R$ is a qF-ring, it follows that $R$ is uniserial. Since $R$ itself is a q-ring, it follows from Jain, Singh and Mohamed (13) that every one-sided large ideal of $R$ is two-sided.

(2) Let us now suppose that $R$ is a uniserial ring all of whose one-sided large ideals are two-sided. Then $R$ is a qc-ring by Theorem 3.3. Now let $I$ be any ideal of $R$. Then $R / I$ is also a qc-ring (see (1), Lemma 3). Since $R$ is uniserial, if follows easily that $R / I$ is a uniserial ring. But again by $(1$, Theorem 11$)$, every uniserial ring which is qc is also a q-ring. Hence $R / I$ is a q-ring. Thus every homomorphic image of $R$ is a q-ring and the theorem follows.

Corollary. Let $R$ be a local Noetherian ring. Then each homomorphic image of $R$ is a q-ring if and only if $R$ is a uniserial ring which is also a duo ring.

Finally, we prove the following theorem.

Theorem 4.13. Let $R$ be a local ring. Then each homomorphic image of $R$ is a q-ring if and only if $R$ is a qc-ring.

Proof. (1) Let us suppose that each homomorphic image of $R$ is a q-ring. In particular, $R$ is a q-ring. It is easy to show that every local q-ring is a duo ring. To show that $R$ is a qc-ring, let us consider a cyclic right $R$-module $M$. Then $M \cong R / I$, where $I$ is a right ideal of $R$. Since $R$ is a duo ring, $I$ is twosided. Then $R / I$ is in fact a factor ring of $R$. This implies that $R / I$ is a selfinjective ring. In other words, $R / I$ is $R / I$-quasi-injective. Then it follows from (1, Lemma 2) that $R / I$ is $R$-quasi-injective. Therefore $R$ is a qc-ring.

(2) Conversely, suppose $R$ is a qc-ring. Then each homomorphic image of $R$ is a qc-ring. Since every qc-ring is also a q-ring (see Koehler (15)), each homomorphic image of $R$ is also a q-ring. This proves the theorem.

\section{Acknowledgement}

The author is grateful to his supervisor, Dr A. D. Sands, for his help in the preparation of this paper. A part of the work on this paper was done at the University of Dundee and the author is thankful to the University for giving a grant which financed this research. 


\section{REFERENCES}

(1) J. AhSAn, Rings all of whose cyclic modules are quasi-injective, Proc. London Math. Soc. (3) 27 (1973), 425-439.

(2) K. Asano, Uber Hauptideal Ringe mit Kettensatz, Osaka J. Math. 1 (1949), 52-61.

(3) H. Bass, Finitistic dimension and a homomological generalization of semiprimary rings, Trans. Amer. Math. Soc. 95 (1960), 466-488.

(4) J.-E. BJöRK, Conditions which imply that subrings of semi-primary rings are semi-primary, J. Algebra, 19 (1971), 384-395.

(5) K. A. Byrd, Some characterizations of uniserial rings, Math. Ann. 186 (1970), 163-170.

(6) W. H. Caldwell, Hypercyclic Rings, Pacific J. Math. 24 (1968), 29-44.

(7) I. S. CoHEN, Commutative rings with restricted minimum Condition, Duke Math. J. 17 (1950), 27-42.

(8) C. FAITH, Lectures on injective modules and quotient rings (Springer-Verlag, New York, 1967).

(9) C. FaITH, On Köthe rings, Math. Ann. 164 (1964), 207-212.

(10) C. FAITh and E. A. WALKer, Direct sum representations of injective modules, J. Algebra 5 (1967), 203-221.

(11) K. R. Fuller, Generalized uniserial rings and their Kupisch series, Math. $Z$. 106 (1968), 248-260.

(12) K. R. Fuller, On direct representations of quasi-injectives and quasiprojectives, Arch. Math. 20 (1969), 495-502.

(13) S. K. JAIN, S. Singh and S. H. Mohamed, Rings in which every right ideal is quasi-injective, Pacific J. Math. 31 (1969), 73-79.

(14) R. E. JoHnson and E. T. WoNG, Quasi-injective modules and irreducible rings, J. London Math. Soc. 36 (1961), 260-268.

(15) ANNe B. KoeHLer, Rings with quasi-injective cyclic modules, Quart. J. Math Oxford (2) 25 (1974), 51-55.

(16) L. S. Levy, Commutative rings whose homomorphic images are self-injective, Pacific J. Math. 18 (1966), 149-153.

(17) S. Mohamed, Rings whose homomorphic images are $q$-rings, Pacific J. Math. 35 (1970), 725-735.

(18) S. Moнамmed, Semi-local q-rings, Indian J. Pure Appl. Math. 1 (1969-1970), 419-424.

(19) A. Pollinghar and A. ZAKs, Some remarks on quasi-Frobenious Rings, J. Algebra 10 (1968), 231-239.

(20) R. C. SHоск, The rings of endomorphisms of a finite dimensional module, Israel J. Math. 11 (1972), 309-314. 
(21) A. ZAKs, Injective dimensions of semi-primary rings, J. Algebra 13 (1969), 73-86.

Department of Mathematics

UNIVERSITY OF ISLAMABAD

ISLAMABAD

PAKISTAN 